\title{
CASO 19-2015:Feohifomicosis nasal por Exophiala dermatidis
}
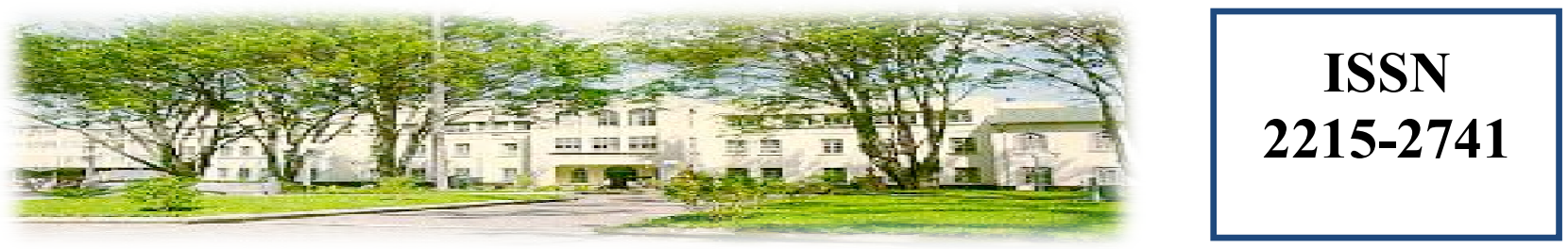

Hospital San Juan de Dios, San José, Costa Rica. Fundado en 1845

$\begin{array}{ll}\text { Recibido: } & 05 / 07 / 2015 \\ \text { Aceptado: } & 15 / 09 / 2015\end{array}$

\author{
Natalia Solís Rojas ${ }^{1}$ \\ Enrique Muñoz Hernández ${ }^{2}$ \\ Manuel Antonio Villalobos Zúñiga ${ }^{3}$
}

1. Médico Residente, Posgrado Infectología, Hospital Calderón Guardia, Caja Costarricense del Seguro Social, natalia1283@yahoo.com

2. Médico Otorrinolaringólogo y Especialista en Cirugía Endoscópica de Senos Paranasales y vértigo, Hospital San Juan de Dios, Caja Costarricense del Seguro Social, drmunozh@gmail.com

3. Médico Asistente Especialista en Medicina Interna e Infectología, , Hospital San Juan de Dios, Caja Costarricense del Seguro Social, manuel701@gmail.com

\section{RESUMEN}

Paciente masculino de 74 años, vecino de Turrubares, domador de caballos, con antecedentes personales patológicos de gastrectomia total, EPOC y osteoporosis. Consulta por tumoración violácea de 6 meses de evolución en fosa nasal izquierda, de crecimiento lentamente progresivo, asociado a epistaxis leve, y rinorrea fétida. Mediante examen físico, cultivo y biopsia se diagnostica feohifomicosis por Exophiala dermatidis. Tras dos meses de resección quirúrgica, se documenta recurrencia de la lesión, por lo que se decide hospitalizar para tratamiento antifungico endovenoso con anfotericina $\mathrm{B}$ deoxycolato $\mathrm{y}$ itraconazol por seis meses. Sin embargo tras dos meses de tratamiento con itraconazol, amerita nueva hospitalización por recaída clínica, realizándose escisión quirúrgica completa y cambiando tratamiento a posaconazol, cursando el paciente posteriormente asintomatico, sin necesidad de nuevas intervenciones terapéuticas.

\section{PALABRAS CLAVE}

Feohifomicosis, Exophiala dermatidis.

\section{ABSTRACT}

A 74-year old male patient, horse breaker from Turrubares, with a medical history of total gastrectomy, COPD and osteoporosis. The patient consulted due to a purplish slow growing tumor of 6 months of evolution in his left nostril. This 
growth was associated with mild epistaxis and foul smelling rhinorrhoea. The medical examination, microbiological cultivation and tissue biopsy showed a Phaeohyphomycosis by Exophiala dermatidis. Two months after surgery, recurrence of the tumor was documented and the patient was hospitalized for endovenous antifungic treatment with deoxycolate anfotericine B and Itraconazol during 6 months. Nevertheless, after two months on itraconazol regimen, the patient was readmitted due to clinical recurrence and a new surgical resection was made. In addition, the treatment was switched to posaconazol. Later the patient was asymptomatic and with no need for new interventions.

\section{KEY WORDS}

Phaeohyphomycosis, Exophiala dermatidis.

\section{ABREVIATURAS}

ORL: Otorrinolaringología, HSJD: Hospital San Juan de Dios

\section{CUADRO CLÍNICO}

Paciente masculino de 74 años, vecino de Turrubares quien se dedica a entrenar caballos en el campo. Presenta como antecedentes personales patológicos una gastrectomía total hace 13 años por cáncer gástrico, EPOC y osteoporosis.

El paciente fue valorado por primera vez en consulta externa del Servicio de ORL del HSJD por historia de seis meses de evolución de tumoración violácea de lento crecimiento en fosa nasal izquierda, asociado a epistaxis leve, y rinorrea fétida, evidenciándose a la exploración física una masa violacea-negruzca de $1,5 \times 1 \mathrm{~cm}$ en fosa nasal izquierda, en mucosa septal, la cual se reseca y se envían muestras al Servicio de Patología y Microbiología. (Figura 1). En retrospectiva se obtiene la información de que el paciente con frecuencia se introduce objetos vegetales en fosas nasales para rascado.

La biopsia posteriormente reportó una Feohifomicosis y en el cultivo se demostró el crecimiento de Wangiella dermatidis (actualmente Exophiala dermatidis). (Figura 2)

El paciente se mantuvo en control en ORL, donde se documenta dos meses después recurrencia de la lesión. Es referido al Servicio de Infectologia, donde se decide hospitalizar, para inicio de tratamiento antifúngico sistémico con anfotericina deoxycolato (500 $\mathrm{mg}$ en total), y se descarta enfermedad diseminada y patología neoplásica predisponente. Se egresa con itraconazol por seis meses.

Sin embargo tras casi dos meses de tratamiento con itraconazol, amerita nuevo internamiento por nuevo crecimiento de la masa, por lo que se decide realizar una biopsia excisional y cambiar a tratamiento a posaconazol por 6 meses.

Luego de 6 meses de la última intervención terapeútica el paciente no ha presentado recurrencias y se encuentra asintomático.

Figura 1. Lesión exofítica en fosa nasal izquierda

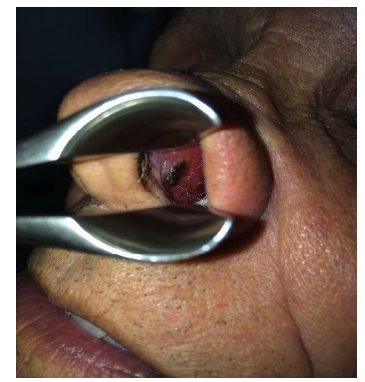

\section{DISCUSIÓN}

La feohifomicosis es el termino general para describir los hongos dematiaceos, o de pigmento oscuro. Son responsables de enfermedades severas tanto en inmunosupresos como inmunocompetentes. ${ }^{(1)}$

Son de distribución mundial y se ubican predominantemente en los suelos. Probablemente la mayoría de los pacientes se expusieron a estos patógenos por inhalación o secundario a herida por trauma penetrante. ${ }^{(1)}$

Debe distinguirse esta patología, de otras condiciones médicas específicas ocasionadas igualmente por hongos dematiaceos, como la cromoblastomicosis y los micetomas. La cromoblastomicosis es originada por un pequeño grupo de hongos que característicamente forman cuerpos escleróticos a nivel tisular, y predominan en zonas tropicales. Los micetomas suelen afectar las extremidades inferiores, observándose granulos micoticos en la microscopía. ${ }^{(1)}$

Se han descrito varias presentaciones clínicas; infecciones superficiales, profundas locales, 
enfermedades alérgicas, neumonías, abscesos cerebrales y la forma diseminada (Tabla I). ${ }^{\text {(2) }}$

La característica distintiva de todas estas especies, es la presencia de melanina en su pared celular, lo cual le imparte un color oscuro a sus conidias, esporas e hifas. Sus hifas típicamente se observan fragmentadas y septadas irregularmente. Sus colonias también se observan de coloración café o negra (Figura 3 ).

Entre los factores de virulencia descritos dentro de los hongos dematiaceos se encuentran los carotenoides y la melanina, los cuales son componentes de su pared celular. La melanina está compuesta por varios monómeros fenólicos, y en menor cuantía por complejos proteínicos, y son parte de la maquinaria de defensa del hongo, pues contrarresta las respuestas inmunológicas del hospedero, bloqueando el efecto de enzimas hidrolíticas, inhibiendo la fagocitosis mediada por receptor, interfiriendo con la producción de oxido nítrico y activando los TLR-4. ${ }^{(3)}$

Los carotenoides sintetizados por estos hongos actúan como "escudos" de protección más que neutralizando los oxidantes tóxicos. ${ }^{(3)}$

Otros componentes de la pared celular como la quitina y el $\beta-1,3-D$-glucano, contribuyen con la morfogénesis estructural, formación de esporas y mantenimiento de la integridad de la pared celular. ${ }^{(3)}$

Figura 2. Histopatología de la feohifomicosis en mucosa nasal A. Epitelio plano estratificado con infiltrado neutrofilico, linfocítico e histiocítico (no granulomatoso) rodeando hifas de pigmento café y un fragment vegetal, B. Tinción de Grocott positiva y C. PAS positiva con visualización de estructuras fúngicas.

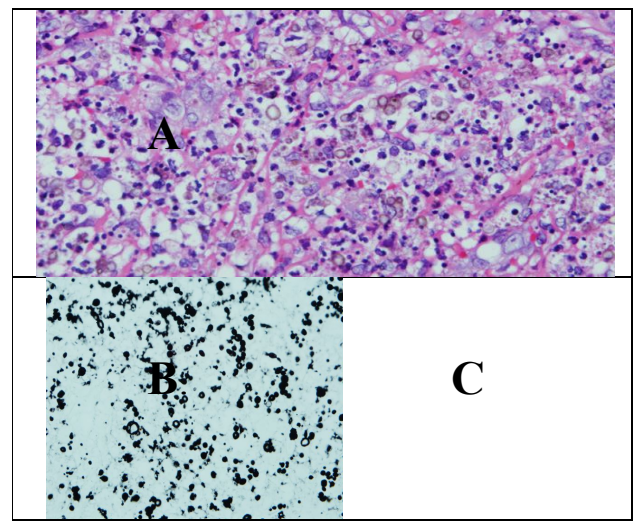

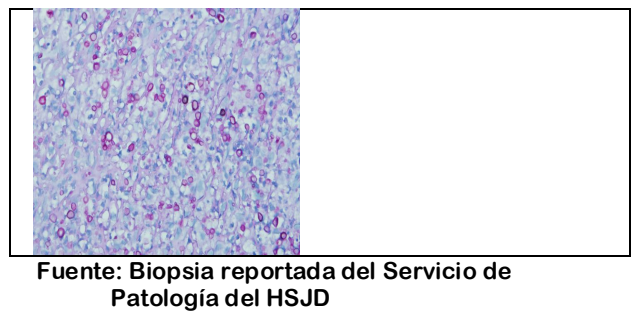

Tabla I. Formas clínicas de Feohifomicosis y su tratamiento

\begin{tabular}{|c|c|c|}
\hline $\begin{array}{l}\text { Presentación } \\
\text { clínica }\end{array}$ & Especie & Tratamiento \\
\hline $\begin{array}{l}\text { Onicomico- } \\
\text { sis }\end{array}$ & $\begin{array}{l}\text { Onychocola } \\
\text { Alternaria }\end{array}$ & $\begin{array}{l}\text { Itraconazol o } \\
\text { Terbinafina o } \\
\text { Agentes tópi- } \\
\cos \end{array}$ \\
\hline $\begin{array}{l}\text { Nódulos } \\
\text { subcutáneos }\end{array}$ & $\begin{array}{c}\text { Exophiala } \\
\text { Alternaria } \\
\text { Phialophora }\end{array}$ & $\begin{array}{l}\text { Cirugía } \\
\text { Itraconazol }\end{array}$ \\
\hline Queratitis & $\begin{array}{c}\text { Curvularia } \\
\text { Bipolaris } \\
\text { Exserohilum } \\
\text { Lasiodiplodia }\end{array}$ & $\begin{array}{l}\text { Natamicina } \\
\text { oftálmica } \\
\text { Itraconazol }\end{array}$ \\
\hline $\begin{array}{l}\text { Enfermedad } \\
\text { alérgica }\end{array}$ & $\begin{array}{c}\text { Bipolaris } \\
\text { Curvularia }\end{array}$ & $\begin{array}{l}\text { Esteroides } \\
\text { Itraconazol }\end{array}$ \\
\hline Neumonía & $\begin{array}{c}\text { Ochroconis } \\
\text { Exophiala } \\
\text { Chaetomium }\end{array}$ & $\begin{array}{l}\text { Anfotericina } \\
\text { Itraconazol }\end{array}$ \\
\hline $\begin{array}{l}\text { Absceso } \\
\text { cerebral }\end{array}$ & $\begin{array}{l}\text { Chladophialop- } \\
\text { hora } \\
\text { Ramichloridium } \\
\text { Ochroconis }\end{array}$ & $\begin{array}{l}\text { Anfotericina } \\
\text { Itraconazol } \\
\text { 5-Fluoracilo }\end{array}$ \\
\hline $\begin{array}{l}\text { Enfermedad } \\
\text { diseminada }\end{array}$ & $\begin{array}{c}\text { Scedosporium } \\
\text { Bipolaris } \\
\text { Exophiala }\end{array}$ & $\begin{array}{l}\text { Anfotericina } \\
\text { Itraconazol } \\
\text { Equinocandi- } \\
\text { nas }\end{array}$ \\
\hline
\end{tabular}
phomycosis. Infect Dis Clin N Am 2006.

Figura 3. A. Colonia con Blastoconodias oscuras fialides cilíndricas con conidias elípticas. B. Cultivo de Exophiala dermatidis (colonia pigmentada)

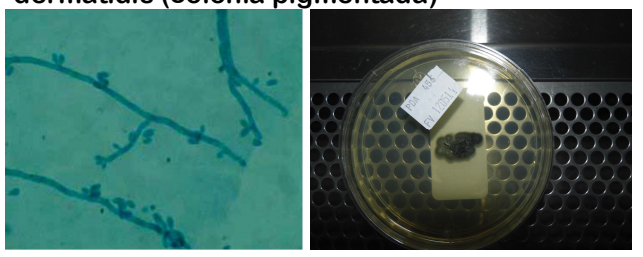

Fuente: A. Juan Pedro Russo, MD, Rocío Raffaeli, MD Stella Maris Ingratta, MD; Patricia Rafti, MD; Silvana Mestroni, MD. 2010. Cutaneous and Subcutaneous Phae- 
ohyphomycosis. SKIN, pag 367. B. Laboratorio de Bacteriología, Sección de Micología, HSJD.

Una característica que puede presentarse en la feohifomicosis es la fase de "levadura-like" descrita en su transición de levadura a hifa. Exclusivamente en el género Exophiala se ha descrito reproducción por gemación. ${ }^{(4)}$ La posibilidad de diseminación hematógena a partir de tejido subcutáneo, depende de esta forma de reproducción, pues la formación de hifas condiciona una afección más localizada, por ende la gran mayoría de infecciones diseminadas van a ser causadas por especies del género Exophiala. ${ }^{(4)}$

Son capaces de producir metabolitos ácidos y alcalinos extracelulares y siderófos, los cuales pueden actuar como factores de virulencia. Se ha demostrado que las altas concentraciones de hierro favorecen la formación de sideróforos a nivel tisular. ${ }^{(4)}$

La enfermedad diseminada por Exophiala, muestra un marcado neurotropismo, se cree secundario a mayor concentración de hierro en $\mathrm{SNC}$ con respecto al suero. ${ }^{(4)}$

La respuesta inmunológica innata corresponde a la primera línea de defensa contra estos hongos. Los macrófagos son las células más importantes en controlar el crecimiento fúngico. ${ }^{(4)}$

Se ha documentado la presencia de gran cantidad de antígenos fúngicos como material homogéneo granular en los macrófagos cutáneos, probablemente por ser las primeras células de defensa, y junto con las células dendríticas fungir como células presentadoras de antígenos. ${ }^{(5)}$

Los neutrofilos son unas de las células fagocíticas predominantes en las lesiones ocasionadas por la feohifomicosis. Su éxito fungicida condiciona menor invasión tisular progresiva y curación. ${ }^{(5)}$

En los especímenes histopatológicos de personas enfermas, la reacción tisular característica es la de tipo granulomatosa, con linfocitos, granulocitos y células gigantes. La asociación con abscesos, sugieren intentos frustrados de fagocitosis de estos hongos, cuya persistencia in situ, es el principal factor determinante de cronicidad y de la alta organización de la reacción inflamatoria observada.

La inmunidad adaptativa manifiesta una alta especificidad antigénica y está compuesta por anticuerpos, células B y Linfocitos Th CD4 y CD8.
El perfil inmunológico predominante Th1/Th2 condicionará la severidad y cronicidad del cuadro. Estudios han revelado que las infecciones severas exhiben un predominio de respuestas de tipo $\mathrm{TH} 2$, con desordenes en la proliferación linfocitica y secretan predominantemente IL-10, y TNF- $\alpha$, sin detectarse IFN- $\gamma$ e IL- 4 . En pacientes con manifestaciones leves predomina la producción de IFN- $\gamma$, bajos niveles de IL-10 y adecuada amplificación linfocitaria. ${ }^{(6)}$ En las formas que se cronifican, el desbalance es a favor de las respuestas Th1, con altos niveles de IL-10 y IL4 , y bajos niveles de IFN- $\gamma .{ }^{(6)}$

No existe evidencia de anticuerpos protectores. Algunos estudios in vitro sugieren que la presencia de anticuerpos anti-melanina podrían inhibir el crecimiento fúngico. ${ }^{(6)}$ Se requiere más de 12 meses para la negativización de las serologías tras haber concluido el tratamiento antifúngico. (6)

Las manifestaciones clínicas más frecuentes de estos hongos son las de tipo alérgico. Se ha relacionado la exposición a Alternaria spp. y Cladosporium spp. con exacerbaciones agudas asmáticas. ${ }^{(2)}$

Es la causante del 6-9\% de las sinusitis crónicas que ameritaran cirugía. ${ }^{(2)}$ Previamente se pensaba que era el Aspergillus spp. el principal hongo responsable de las sinusitis crónica, sin embargo actualmente se sabe que los hongos dematiaceos son la principal causa. ${ }^{(2)}$ Las especies más comúnmente aisladas son Alternaria, Bipolaris, y Curvularia.

Los criterios diagnósticos propuestos son: i) pólipos nasales, ii) mucina alérgica, con cristales de Charcot-Leyden, y eosinófilos, iii) hifas en la mucosa sin datos de invasión tisular, iv) pruebas cutáneas positivas a alérgenos fúngicos, y v) tomografías con evidencia de áreas de hiperatenuación central dentro de la cavidad sinusoidal. No todos los criterios son necesarios para la realización del diagnóstico, ${ }^{(2)}$ sin embargo es fundamental la presencia de mucina alérgica, con o sin un cultivo positivo. La mayoría de los cuadros resuelven con la intervención quirúrgica, sin embargo en algunos casos ha sido necesario el uso de esteroides sistémicos. En casos refractarios el uso de antifungicos si ha mostrado mejorar el pronóstico. ${ }^{(2)}$

La micosis broncopulmonar alérgica (ABPM) predomina en asmáticos y pacientes con fibrosis quística ${ }^{(7)}$ sobretodo los géneros Bipolaris o Curvularia. ${ }^{(7)}$ La terapia es con esteroides sisté- 
micos, con un des escalonamiento a los dos o tres meses.

Las infecciones superficiales involucran sólo tejidos queratinizados como las uñas de pies y manos y el estrato córneo. Las consecuencias son especialmente de tipo cosmético. Son causas raras de onicomicosis, siendo Alternaria, Scopulariopsis y Neoscytalidium, causantes de la mayoría de los casos. ${ }^{(8)}$ La tiña nigra es una manifestación infrecuente, caracterizándose por la presencia de una macula pigmentada, usualmente en palmas y plantas. La mayoría de los casos reportados son causados por Hortaea werneckii, con el antecedente de visita reciente a playas arenosas.

La afección subcutánea corresponde a la mayoría de casos descritos por estos hongos oscuros, siendo la Alternaria el agente etiológico más frecuente ${ }^{(8)}$, y Exophiala y Phialophora los siguientes en frecuencia.

Muchos de los pacientes son inmunocompetentes, viven en zonas rurales, y son campesinos o jardineros con traumas menores por cuerpos vegetales. Otro grupo de riesgo son los pacientes trasplantados.

Las lesiones típicamente ocurren en áreas corporales expuestas, como lesiones quísticas o papulares aisladas, usualmente indolentes, de semanas a meses de evolución llegando a convertirse en masas grandes. El mayor riesgo de diseminación ocurre en los pacientes severamente inmunosupresos y ocasionalmente la infección puede comprometer las articulaciones y hueso, recomendándose en estos casos terapia antifúngica prolongada de 6 a 24 meses. ${ }^{(9)}$

La terapéutica depende del status inmunológico del paciente y la extensión de la lesión, se recomienda la terapia anti fúngica sistémica en conjunto con la cirugía, particularmente en pacientes inmunocomprometidos, para prevenir la forma diseminada. La escisión quirúrgica como único tratamiento, ha sido exitosa en algunos trasplantados de órgano sólido ${ }^{(10)}$, inclusive en pacientes con múltiples lesiones, donde la resección puede ser más complicada. Las recurrencias pueden ocurrir varios meses o años tras el término del tratamiento, por ende, es indispensable el seguimiento clínico y la educación al paciente.

La presentación pulmonar, es una complicación casi exclusiva de pacientes inmunosupresos o con patología pulmonar subyacente y puede ser ocasionada por varios gérmenes como $\mathrm{S}$. prolificans, C. bantiana, Chaetomium spp., Ochroconis gallopava, Exophiala spp., Alternaria, Cladophialophora boppii, F. pedrosoi, L. theobromae, Aureobasidium pullulans, Curvularia spp., Sarcinosporin inkin, y P. verrucosa. Se pueden manifestar clínicamente como una neumonía, nódulos pulmonares solitarios asintomáticos o lesiones endobronquiales generando hemoptisis. La mortalidad alcanza hasta un $40 \% .{ }^{(11)}$ En el caso de nódulos solitarios pulmonares la resección quirúrgica parece ser suficiente.

Usualmente la infección del Sistema Nervioso Central, es una manifestación fatal, con mayor frecuencia en pacientes inmunocompetentes, siendo el absceso cerebral el hallazgo más frecuente. El agente más comúnmente aislado es $\mathrm{C}$. bantiana. Se cree que se debe a la diseminación hematogena de un foco pulmonar sub clínico.

La terapeútica es complicada, y no existe evidencia clara de las mejores alternativas de manejo. Probablemente la mejor posibilidad de sobrevida en estos pacientes se consiga con la combinación de anfotericina, flucitosina e itraconazole. Otras alternativas de manejo con voriconazole y posaconazole se han reportado, con alguna tasa de éxito. Lo que sí parece ser consistente es que la escisión completa del absceso cerebral garantiza un mejor pronóstico vs la aspiración parcial de la lesión.

La enfermedad diseminada es la forma más infrecuente de manifestación clínica, de predominio en inmunosupresos. Los hemocultivos están positivos en el $50 \%$ de los casos. La mortalidad es del $70 \%$ aproximadamente, y la posibilidad de sobrevida depende de la recuperación inmunológica. Dada la infrecuencia del cuadro, no hay una recomendación clara de tratamiento. En el caso de Scedosporium prolificans, el manejo se complica, pues este germen es generalmente resistente a la mayoría de antifungicos disponibles. Algunos han utilizado factor estimulante de colonias o infusión de leucocitos junto con antifúngicos como tratamiento. ${ }^{(12)}$

No existen pautas establecidas de tratamiento. Los medicamentos que han mostrado actividad in vitro son el itraconazol, el voriconazol, y el posaconazol. Exceptuando por las especies Scedosporium prolificans y Scopulariopsis brumptii, itraconazol y voriconazol han demostrado buena actividad contra la mayoría de los hongos dematiaceos, siendo la MIC generalmente $\leq$ $0,125 \mathrm{mg} / \mathrm{mL}$. ${ }^{(13)}$

El fluconazol carece de actividad contra estos patógenos y el uso de Ketoconazol es muy limi- 
tado por sus efectos adversos. La anfotericina sola, o en combinación con flucitosina, voriconazol o itraconazol no ha sido completamente satisfactoria ${ }^{(14)}$. Sin embargo la combinación de anfotericina, itraconazol y flucitosina han demotrado mejoría en la sobrevida en algunos casos reportados. ${ }^{(15)}$ La terapia combinada se recomienda en abscesos cerebrales cuando no hay posibilidad de intervención quirúrgica, $y$ en infecciones diseminadas en pacientes inmunocomprometidos. $^{(16)}$

Dada la buena penetración en sistema nervioso central, el uso de voriconazol está recomendado.

(17) Posaconazol también ha demostrado éxito en las formas cerebral y diseminada. ${ }^{(18)}$

Las equinocandinas pueden utilizarse, sin embargo cuentan con poca evidencia de actividad en hongos dematiaceous.

\section{CONCLUSIÓN}

Se ha presentado el infrecuente caso de una feohifomicosis por Exophiala dermatidis en un paciente masculino de 74 años domador de caballos en la zona rural de Costa Rica, en la mucosa nasal izquierda; donde luego de una primera recaída clínica, el tratamiento con resección quirúrgica completa, anfotericina $\mathrm{B}$ y posaconazol ha sido curativo.

\section{BIBLIOGRAFÍA}

1. McGinnis MR. Chromoblastomycosis and phaeohyphomycosis: new concepts, diagnosis and mycology. J Am Acad Dermatol 1983; 8:1-16.

2. Sanjay G Revankar MD. Phaeohyphomycosis. Infect Dis Clin N Am 20 (2006); 609-220.

3. Nosanchuk JD Casadevall A. 2003. The contribution of melanin to microbial pathogenesis. Cell. Microbiol. 5:203-223.

4. De Hoog GS. 1993. Evolution of black yeasts: possible adaptation to the human host. Antonie Van Leeuwenhoek 63:105-109.

5. Romani L. 2011. Immunity to fungal infections. Nat. Rev. Immunol. 11:275-288.

6. Seyedmousavi S Netea MG Mouton JW Melchers WJ Verweij PE de Hoog GS. Black yeasts and their filamentous relatives: principles of pathogenesis and host defense. Clin Microbiol Rev. 2014 Jul;27(3):52742

7. Agarwal R. 2009. Allergic bronchopulmonary aspergillosis. Chest 135:805-826.
8. Halwig, J. M., D. A. Brueske, P. A. Greenberger, R. B. Dreisin, and H. M. Sommers. 1985. Allergic bronchopulmonary curvulariosis. Am. Rev. Resp. Dis. 132:186-188.

9. Chabasse D Bievre C Legrand E et al. Subcutaneous abcesss caused by Pleurophopmosis lignicola first case. J Med Vet Mycol 1995; 33: 415-417.

10. Diaz M Puente R Trevino MA. Response of long running Alternaria alternata response to floconazole Lancet 1990; 336: 513.

11. Ara M Aspiroz A Zavallos V et al. Relapse of cutaneous Alternaria infectoria in a renal trasplant recipient after 2 years. Act Derm Venereol 2006; 86: 154-155.

12. Crichlow D Enrile T Memon M. Cerebellar abscess due to Cladosporium trichoides (bantianum): case report. Am. J. Clin. Pathol; 1973; 60:416-421.

13. Whyte M Irving P O'Regan, M Nissen, D Siebert Labrom R. Disseminated Scedosporium prolificans infection and survival of a child with acute lymphoblastic leukemia. Pediatr. Infect. Dis. J 2005; 24:375377.

14. McGinnis MR Pasarell L. In vitro testing of susceptibilities of filamentous ascomycetes to voriconazole, itraconazole, and amphotericin $B$, with consideration of phylogenetic implications. J Clin Microbiol 1998;36:2353-5.

15. Zeng JS Sutton DA Fothergill AW Rinaldi MG Harrak MJ de Hoog GS. Spectrum of clinically relevant Exophiala species in the United States. J. Clin. Microbiol 2007 45:3713-3720.

16. Revankar SG, Sutton DA, Rinaldi MG. 2004. Primary central nervous system phaeohyphomycosis: a review of 101 cases. Clin. Infect. Dis. 38: 206-216. 17 Chowdhary A Meis JF Guarro J et al. ESCMID and ECMM joint clinical guidelines for the diagnosis and management of systemic phaeohyphomycosis: diseases caused by black fungi. Clin. Microbiol. Infect 2014; 20(Suppl 3):47-75.

18. Al-Abdely HM Alkhunaizi AM Al-Tawfiq JA Hassounah M Rinaldi MG Sutton DA. Successful therapy of cerebral phaeohyphomycosis due to Ramichloridium mackenziei with the new triazole posaconazole. Med. Mycol 2005; 43:91-95.

\section{CONFLICTO DE INTERÉS Y/O AGRADE- CIMIENTOS}

Los autores declaran que no existió ningún conflicto de interés en el presente reporte. 